\title{
Nonlinear Regenerative Dynamics Analysis of the Multicutter Turning Process
}

\author{
A. M. Gouskov, M. A. Guskov, D. D. Tung, G. Y. Panovko
}

This work presents nonlinear dynamics modeling results for an investigation of continuous cut stability in multicutter turning. The dynamics modeling of the multicutter turning process is carried out through the complete mathematical model of nonlinear dynamics. The dynamic stability of the system is estimated through the possibility of self-oscillations generation (Poincaré - Andronov - Hopf bifurcation) of the cutters with lobes of the stability diagram. This paper analyzes the relationship of the axial offset and the cutter angular position for compensation of the system parameters. As a result, the analysis of the influence of the technological system parameters on the chip thickness, their cross-sectional shape and the stability of the system is carried out.

Keywords: multicutter turning, dynamics, modeling, bifurcation analysis, steady cutting stability conditions

Received January 07, 2019

Accepted May 28, 2019

This work was supported by the Russian Science Foundation, project No 18-19-00708.

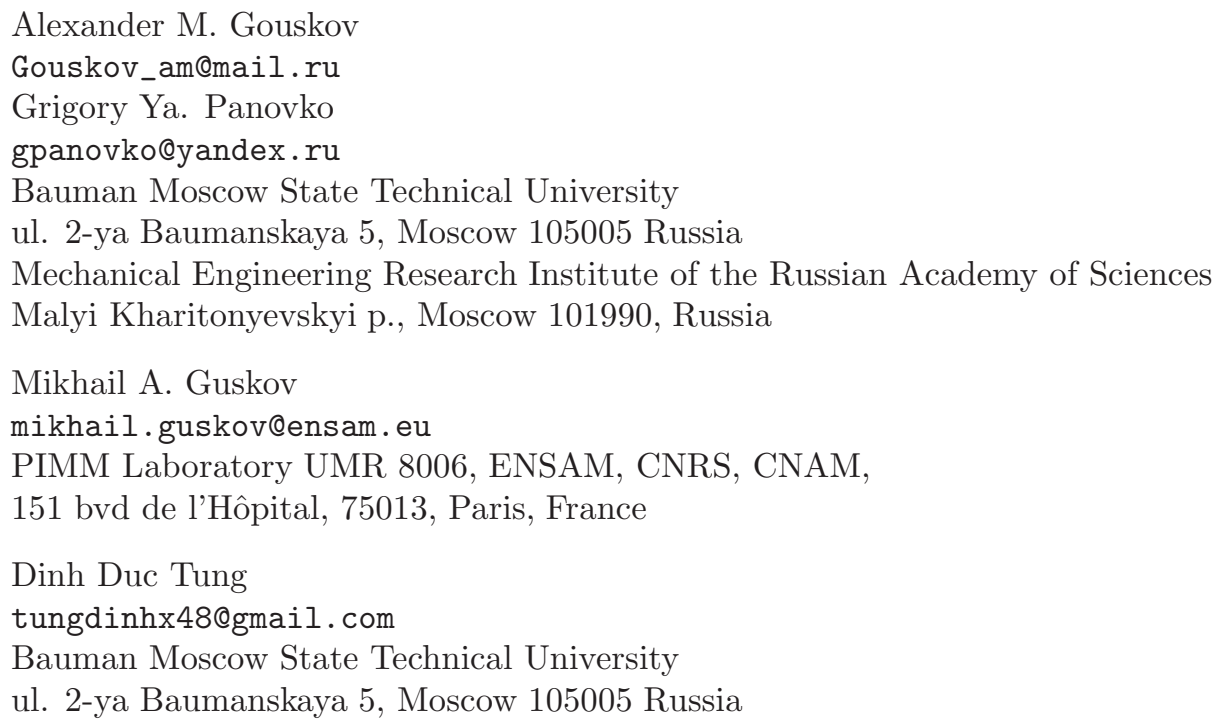




\section{Introduction and problem formulation}

One of the methods of increasing the productivity of the turning process is the use of multiple cutter systems, in which the effective depth of cut in one pass can be increased, the cutting forces can be partially balanced (especially in the transversal direction, which is particularly important for thin-walled workpieces), cutting operations can be combined (e.g., raw and finishing), and the processing time can be substantially reduced. Several works have addressed multiple cutter turning systems in recent years $[2,4,8,9,10,11,12,13,19,20]$.

Under certain conditions, the cutting with constant depth can become dynamically unstable. The problems of oscillations arising during machining has been discussed in numerous works $[6$, $15,17,18,21]$. One of the reasons for the loss of stability are nonlinear phenomena underlying the cutting and friction forces (in particular, ploughing on the flank face of the cutter), depending on the relative motion in the cutting interaction zone, and potentially leading to the interrupted cut (chip fragmentation) $[5,16,17]$. Another class of important causes of instabilities are the regeneration of cut surfaces (i.e., cutting the surfaces formed during the previous pass of the cutter), workpiece flexibility, temperature effects etc. $[1,3,6,15]$. One of the important problems in the technological processes of turning is the self-excitation of the cutting tool vibration. In some cases, tool vibrations can have a positive effect on the machining process: they allow cutting forces to be reduced. But in most cases, the self-excitation of vibration is an extremely harmful phenomenon, which reduces the quality and accuracy of vibration-free turning.

\section{Multiple cutter turning dynamics model}

This paper discusses the longitudinal turning scheme of a round workpiece with several cutters simultaneously (Fig. 1).

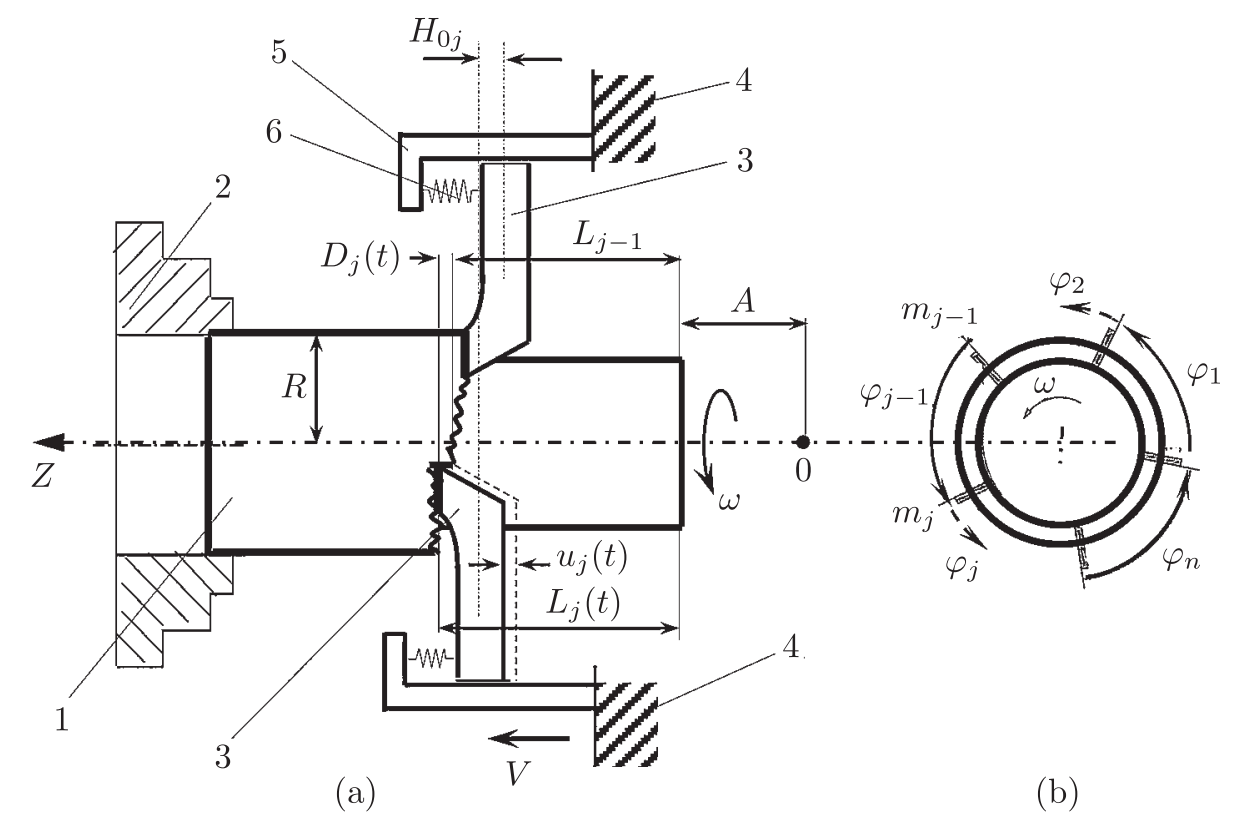

Fig. 1. Multiple cutter process model.

Workpiece (1) is rigidly fixed in the spindle (2) of the lathe (Fig. 1a). Cutters (3) are located around the circumference of the part at an angle $\varphi_{j}, j=\overline{1, n}$ ( $n$ is the number of 
cutters), moreover, $\sum_{j=1}^{n}\left(\varphi_{j}\right)=2 \pi$ (Fig. 1b). Each subsequent cutter is installed with a certain axial displacement of $\mathrm{H}_{0 j}$ in the machining plane relative to the adjacent $(j+1)$ th cutter. All the cutters are mounted on a common support (4), moving at a constant speed $\mathrm{V}$ along the axis of the part. The part rotates around its longitudinal axis with a constant angular velocity $\omega$. The cutters have coupled dynamics - they interact with each other through the formed surface. That is, the $j$ th cutter interacts with the surface at time $t$ formed by the "leading ahead" $(j+1)$ th cutter at time $t-\varphi_{j} / \omega$.

In this work we consider multicutter vibration with axial compliance of the cutters. Depending on the stiffness and relative positioning, different oscillation and chip formation patterns take place. This paper is a development of studies conducted in $[8,9,10-13]$. Unlike our previous works, the main goal of this study is to identify the effect of technological parameters of cutting (depth of cut, cutting speed, angular speed of the part) and the installation axial and / or angular displacement of the cutters on the stability of the continuous (vibration-free) cutting mode. This paper analyzes the nonlinear dynamics and stability of the process of turning a part along the axis of rotation. Conditions will be formed for mutual compensation of angular and axial displacements that do not lead to a change in the boundaries of areas of stability of continuous cutting. Determining the limits of stability, the passage of which leads to a rigid excitation of vibrations (the subcritical Poincaré - Andronov-Hopf bifurcation [6]) makes it possible to reasonably designate the cutting tool installation parameters and the technological processing parameters at which the risk of tool vibration is minimized.

The present work is focused on the dynamics and stability of multicutter turning as a function of cutters' positions and of process parameters (cut velocity), with the regenerative effect considered. Thus, the conditions of auto-excitation of oscillations and of steady cut are analyzed.

The paper is organized as follows. First, a model for the dynamics of a multiple cutter turning system is elaborated for an arbitrary number of cutters, in the form of a system of delayed differential-algebraic equations (DAE). Then a specific case of two cutters is examined in detail, and the steady continuous cut stability conditions are analyzed; a methodology for SLD construction is proposed. Finally, a time domain simulation approach is developed for this problem, based on $\varepsilon$-embedding.

Every cutter is considered as a rigid body, fixed on the carrier via a holder with finite axial stiffness. We thus have a dynamical system with one degree of freedom (DOF) per cutter, corresponding to its axial displacement, and the cutting forces will be taken to be axial. It can be observed that the analysis that follows below can also be applied to the case of a rotating tool carrier and of the axial feed motion of the part. The mathematical model of nonlinear dynamics of the process under study can be described by a system comprising three groups of relationships $[6,7,8,12]$ :

- equation of motion;

- cutting law;

- generation of new surfaces.

The equations of motion for the axial vibrations of the cutters can be written as follows $[11,12]$ :

$$
m_{j} \ddot{u}_{j}=-d_{j} \dot{u}_{j}-k_{j} u_{j}+F_{j}\left(t, h_{j}\right) ; \quad j=\overline{1, n},
$$

where $m_{j}$ is the mass of the $j$ th cutter, $d_{j}$ and $k_{j}$ are, respectively, the damping and stiffness coefficients of the $j$ th tool holder, $u_{j}(t)$ is the axial displacement (vibrations) of the $j$ th cutter

RUSSIAN JOURNAL OF NONLINEAR DYNAMICS, 2019, 15(2), 145-158 
with respect to its nominal (quasi-steady) state, and $h_{j}(t)$ is the current value of the thickness being removed by the $j$ th cutter.

Cutting forces in the axial direction acting on the $j$ th cutter are governed by a fractional rational function $[8,11]$ :

$$
F_{j}(t)=K_{0} h_{j}(t) \frac{c+r h_{j}(t)}{c+h_{j}(t)} ; \quad j=\overline{1, n},
$$

where $K_{0}=\gamma \sigma_{L} B$ is the apparent statical stiffness coefficient, $\sigma_{L}$ is the characteristic stress value for the workpiece material, $\mathrm{B}$ is the cut width, and $\gamma, r, c$ are the coefficients determined by experiment $([\gamma]=1,[r]=1,[c]=m)$. Other existing models of cutting forces that take into account various technological parameters of the process (for example, the effect of cutting speed, application of coolant, etc.) are actually taken into account in the values of the experimental coefficients used in this work. It should be emphasized that the law (2.2) used here, unlike those used traditionally, for example, the exponential cutting law - $F(h)=a h^{b}$ (see, for example, $[2,3,6])$ - with two experimental constants $a, b<1$, has a finite derivative at zero: static cutting rigidity at zero $d F /\left.d h\right|_{h=0}$ must be finite. For power dependence $d F /\left.d h\right|_{h=0}=$ $=\left.a b h^{b-1}\right|_{h \downarrow 0} \longrightarrow \infty$. For the law used in this work (2.2) $d F /\left.d h\right|_{h=0}=K_{0}<\infty$. This is important for the numerical implementation of intermittent cutting, where the thickness of the removed layer is zeroed during the cutting process. The removed material thickness $h_{j}(t)$ under the $j$ th cutter depends on the state of the surface after the passage of the previous, $(j-1)$ th cutter. The location of the surface generated by the $j$ th cutter is a function of time and can be defined by the distance to the free end of the part. Thus, the amount of matter removed during this surface generation is comprised between the actual $j$ th cutter position at current time $\mathrm{t}$ and the position of the previous cutter at time $t-t_{j-1}$, where $t_{j-1}=\varphi_{j-1} / \omega$ is the delay between cutters $(j$ and $(j-1))$. These features can be described by the following relationships, based on $[7,8,9,15]:$

$$
\left\{\begin{array}{l}
D_{j}(t)=V t-u_{j}(t)-L_{j-1}\left(t-t_{j-1}\right)+A-H_{0 j}, \\
h_{j}(t)=\max \left[0, D_{j}(t)\right], \\
L_{j}(t)=L_{j-1}\left(t-t_{j-1}\right)
\end{array}\right.
$$

where (see Fig. 1a) $D_{j}(t)$ is the distance from the $j$ th cutting edges to the surface it is processing (the surface left after the passage of the previous, $(j-1)$ th, cutter at time $t-t_{j-1} ; L_{j}(t)$ is the distance from the free end of the workpiece to the surface being left by the $j$ th cutter; $u_{j}(t)$ are the oscillations of the $j$ th cutter (in the axial direction) as the deviation from the nominal (quasi-static) state; $A$ is the distance from the initial position of the cutting edge of the first cutter to the free end of the workpiece; $H_{0 j}$ is the axial offset of the $j$ th cutter with respect to the first one.

Equations (2.1)-(2.3) enable one to take into account the regenerative vibration excitation mechanism in the system. The surface being currently generated at time $t$ results from the preceding evolution of the cutter's position. These equations form a system of differentialalgebraic equations with multiple delays and describe the dynamics of the multiple cutter turning with surface regeneration.

The system (2.1)-(2.3) can be nondimensionalized using the distance scale factor $X_{*}$ equal to the feed per turn $h_{0}$, time scale factor $T_{*}=\sqrt{\sum_{i=1}^{n}\left(T_{i}^{2} / n\right)}, T_{i}=2 \pi \sqrt{m_{j} / k_{j}}, T_{i}$ is the free 
oscillation period for the $j$ th cutter, force scale factor $F_{*}=K_{0} h_{0}$. Then, when all the cutters are identical and the conditions under which the cutters are fastened are the same $\left(m_{i}=m, k_{i}=\right.$ $\left.=k, d_{i}=d, T_{i}=T_{*}\right)$, Eqs. (2.1)-(2.3) in dimensionless form become

$$
\left\{\begin{array}{l}
\Delta_{j}(\tau)=\tau / \rho-\xi_{j}(\tau)-\Lambda_{j-1}\left(\tau-\tau_{j-1}\right)+A-\mathrm{H}_{0 j}, \quad \sum_{j=1}^{n}\left(\tau_{j}\right)=\rho, \\
\eta_{j}(\tau)=\max \left[0, \Delta_{j}(\tau)\right], \\
0=-\Lambda_{j}(\tau)+\Lambda_{j-1}\left(\tau-\tau_{j-1}\right), \\
\xi_{j}^{\prime \prime}=-4 \pi \varsigma \xi_{j}^{\prime}-4 \pi^{2} \xi_{j}+4 \pi^{2} \kappa \Pi_{j}, \quad j=\overline{1, n} .
\end{array}\right.
$$

Here the dimensionless parameters are defined as follows:

$$
\begin{array}{r}
\xi_{j}=\frac{u_{j}}{h_{0}}, \quad \varsigma=\frac{d}{2 \sqrt{m k}}, \quad \kappa=\frac{K_{0}}{k}, \eta_{j}=\frac{h_{j}}{h_{0}}, \quad \eta_{*}=\frac{c}{h_{0}}, \quad \Lambda_{j}=\frac{L_{j}}{h_{0}}, \\
\Delta_{j}=\frac{D_{j}}{h_{0}}, \quad \rho=\frac{2 \pi}{\omega T_{*}}, \quad A=\frac{A}{h_{0}}, \quad \mathrm{H}_{0 j}=\frac{H_{0 j}}{h_{0 j}}, \quad \Pi_{j}=\frac{F_{j}}{K_{0} h_{0}} .
\end{array}
$$

Here $\rho$ is the ratio of the cutters' eigenfrequency to the workpiece rotation frequency (thus $1 / \rho$ would represent the dimensionless cutting speed); $\kappa$ is the relative static apparent stiffness of cutting, in this case identical for every cutter-workpiece interaction; $\Pi_{j}$ is the dimensionless axial component of the cutting force. The system of equations (2.4) is a complete dynamical model of multiple cutter turning which includes the possibility of discontinuous cutting and takes into account the initial tool entry into the part. In this system the unknowns are dimensionless displacements and cut thickness values for each cutter: $\left\{\Delta_{j}, \eta_{j}, \Lambda_{j}, \xi_{j} ; j=\overline{1, n}\right\}$.

Functions $\Lambda_{j}$ depend on delayed argument. Thus, their definition requires an initial set: $\left\{\left.\xi_{j}(\tau)\right|_{\tau=0}=\xi_{j 0},\left.\xi_{j}^{\prime}(\tau)\right|_{\tau=0}=\xi_{j 0}^{\prime},\left.\Lambda_{j}(\tau)\right|_{\tau \in\left[-\tau_{j-1}, 0\right)}=\Lambda_{j 0}(\tau)\right\}$

In this work we will assume $\Lambda_{j}(\tau)=0, \tau<0$, which would correspond to a perfectly flat surface of the part before machining. The initial functions $\Lambda_{j}$ in (2.4) should fulfill the closure of the surface: $\left\{\Lambda_{j 0}(0)=\Lambda_{(j-1) 0}\left(-\tau_{j-1}\right), \Lambda_{(j-1) 0}(0)=\Lambda_{j 0}\left(-\tau_{j}\right)\right\}$. These conditions provide a connection between the $n$th and the first cutter, due to the processed surface.

\section{Two-cutter turning}

In the particular case of two-cutter turning, the circumference of the workpiece cross-section is divided into two unequal parts (Fig. 2), defined by angles $\varphi_{1}=\pi-\Delta_{\varphi}$ and $\varphi_{2}=\pi+\Delta_{\varphi}$, with $\Delta_{\varphi}$ the difference of each angle with $\pi$ (Fig. $2 \mathrm{~b}$ ). The delay for each cutter reads

$$
\tau_{1}=(\rho / 2 \pi) \varphi_{1}, \quad \tau_{2}=(\rho / 2 \pi) \varphi_{2},
$$

where $\rho=\tau_{1}+\tau_{2}=2 \pi /\left(\omega T_{*}\right)$ is the dimensionless period of revolution of the part, inversely proportional to the cutting speed.

Then Eqs. (2.4) can be rewritten as

$$
\left\{\begin{array}{l}
\Delta_{1}(\tau)=\tau / \rho-\xi_{1}(\tau)-\Lambda_{2}\left(\tau-\tau_{2}\right)+A \\
\Delta_{2}(\tau)=\tau / \rho-\xi_{2}(\tau)-\Lambda_{1}\left(\tau-\tau_{1}\right)+A-\mathrm{H}_{0}, \\
\eta_{j}(\tau)=\max \left[0, \Delta_{j}(\tau)\right] ; \quad \Pi_{j}=\eta_{j} \frac{\eta_{*}+r \eta_{j}}{\eta_{*}+\eta_{j}} \\
\Lambda_{1}(\tau)=\Lambda_{2}\left(\tau-\tau_{2}\right)+\eta_{1}(\tau) ; \Lambda_{2}(\tau)=\Lambda_{1}\left(\tau-\tau_{1}\right)+\eta_{2}(\tau), \\
\xi_{j}^{\prime \prime}=-4 \pi \varsigma \xi_{j}^{\prime}-4 \pi^{2} \xi_{j}+4 \pi^{2} \kappa \Pi_{j} ; \quad j=1,2
\end{array}\right.
$$

with the initial conditions $\left\{\Lambda_{10}(0)=\Lambda_{20}\left(-\tau_{2}\right), \Lambda_{20}(0)=\Lambda_{10}\left(-\tau_{1}\right)\right\}$. 

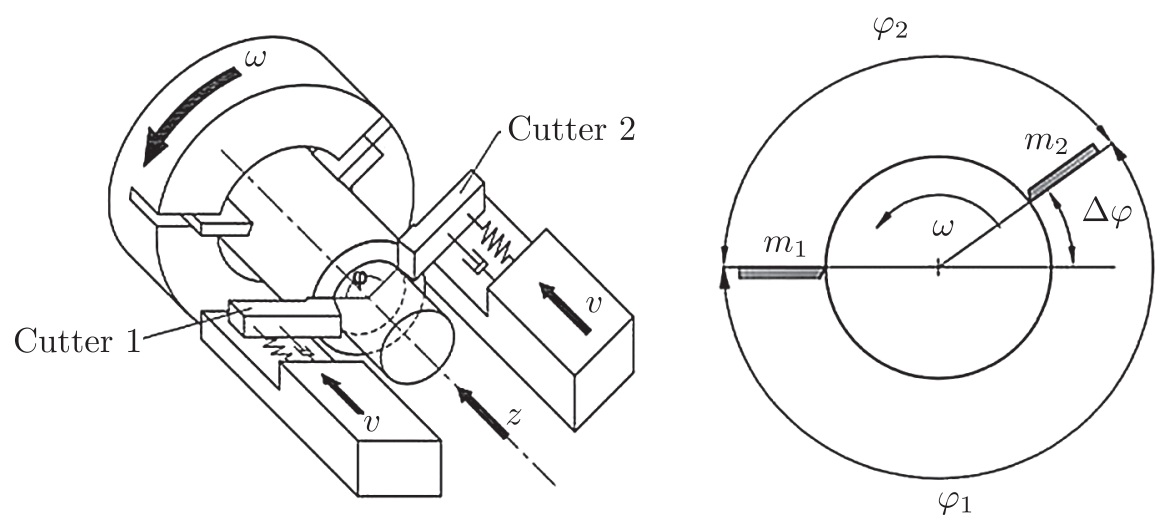

Fig. 2. Schematic of the two-cutter turning.

Two parameters can be high-lighted as specifically relevant to the two-cutter aspect of the analyzed system: the axial offset $H$ and delay ratio $\alpha=\varphi_{1} / \varphi_{2}=\tau_{1} / \tau_{2}$. These parameters will be used in the following analyses.

\subsection{Steady continuous cut conditions}

In the case of steady continuous cutting process, the cut thickness is equal for both cutters, i.e., $\eta_{j}=\Delta_{j} \geqslant 0 ; j=1,2$, as one can infer from (3.2)

$$
\left\{\begin{array}{l}
\eta_{1}(\tau)=\Delta_{1}(\tau)=\tau_{2} / \rho-\xi_{1}(\tau)+\xi_{2}\left(\tau-\tau_{2}\right)+\mathrm{H} \\
\eta_{2}(\tau)=\Delta_{2}(\tau)=\tau_{1} / \rho-\xi_{2}(\tau)+\xi_{1}\left(\tau-\tau_{1}\right)-\mathrm{H}
\end{array}\right.
$$

After substituting (3.3) into the general system (3.2), we obtain the equations of motion in their classical form:

$$
\xi_{j}^{\prime \prime}=-4 \pi \varsigma \xi_{j}^{\prime}-4 \pi^{2} \xi_{j}+4 \pi^{2} \kappa \Pi_{j}, \quad \Pi_{j}=\eta_{j} \frac{\eta_{*}+r \eta_{j}}{\eta_{*}+\eta_{j}}, \quad j=1,2
$$

Equations (3.4) can be used for the stability analysis of the steady continuous cut at constant cut thickness $\eta_{j 0}=$ const $_{j}$. According to Eqs. (3.3) and (3.4), the continuous cut with constant thickness is only possible when the cutter positions are constant: $\xi_{j 0}=$ const $_{j}$. Thus, from (3.3) and (3.4), it follows:

$$
\left\{\begin{array}{l}
\eta_{10}=\tau_{2} / \rho-\xi_{10}+\xi_{20}+\mathrm{H}, \quad \eta_{20}=\tau_{1} / \rho-\xi_{20}+\xi_{10}-\mathrm{H} \\
\Pi_{j 0}=\eta_{j 0} \frac{\eta_{*}+r \eta_{j 0}}{\eta_{*}+\eta_{j 0}}, \quad \xi_{j 0}=\kappa \Pi_{j 0}, \quad j=1,2 .
\end{array}\right.
$$

And one can notice that

$$
P_{j}=\left.\frac{d \Pi_{j}}{d \eta_{j}}\right|_{\eta_{j}=\eta_{j 0}}=r+\frac{\eta_{*}^{2}(1-r)}{\left(\eta_{*}+\eta_{j 0}\right)^{2}}, \quad j=1,2
$$

represent the effective cutting stiffness coefficients, depending on $\eta_{0 j}$, in particular, $p_{j \eta_{0 j}=0}=$ $=1,\left.p_{j}\right|_{\eta_{0 j} \rightarrow \infty}=r ; r \leqslant p_{j} \leqslant 1$. 


\subsection{Stability analysis for steady continuous cut}

For a given set of parameters of nominal process $\tau_{1}, \tau_{2}, \rho, \eta_{*}, r, \kappa, \mathrm{H}$ the system (3.5) provides a steady solution $\eta_{10}, \eta_{20}, \Pi_{10}, \Pi_{20}, \xi_{10}, \xi_{20}$ with stiffness coeffitients $p_{1}, p_{2}$.

One can analyze the perturbed motion in the vicinity of the nominal solution by means of the equations in variations:

$$
\left\{\begin{array}{l}
\delta \xi_{1}^{\prime \prime}+4 \pi \varsigma \delta \xi_{1}^{\prime}+4 \pi^{2} \delta \xi_{1}=4 \pi^{2} \kappa p_{1}\left(-\delta \xi_{1}(\tau)+\delta \xi_{2}\left(\tau-\tau_{2}\right)\right), \\
\delta \xi_{2}^{\prime \prime}+4 \pi \varsigma \delta \xi_{2}^{\prime}+4 \pi^{2} \delta \xi_{2}=4 \pi^{2} \kappa p_{2}\left(-\delta \xi_{2}(\tau)+\delta \xi_{1}\left(\tau-\tau_{1}\right)\right) .
\end{array}\right.
$$

For fixed values $\tau_{1}, \tau_{2}, \eta_{*}, r, \mathrm{H}$ the coefficients $p_{1}, p_{2}$ are determined from relations (3.6).

The solution of the linear system (3.7) is sought in the form $\delta \xi_{j}=C_{j} \exp (\lambda \tau)$, which after being substituted into (3.7) yields the following characteristic equation:

$$
\begin{array}{r}
P(\lambda ; \varsigma, \rho, \kappa)=\left[\lambda^{2}+4 \pi \varsigma \lambda+4 \pi^{2}\left(1+\kappa p_{1}\right]\left[\lambda^{2}+4 \pi \varsigma \lambda+4 \pi^{2}\left(1+\kappa p_{2}\right]-\ldots\right.\right. \\
\ldots-16 \pi^{4} \kappa^{2} p_{1} p_{2} \exp (-\lambda \rho)=0 .
\end{array}
$$

The characteristic equation (3.8) allows one to find, for each value of $\lambda$, the critical value of the static stiffness coefficient as a function of the revolution period $\rho$. It is worth noting that in the dynamical system for $(3.8)-P(0 ; \varsigma, \rho, \kappa)=16 \pi^{4}\left(1+\kappa p_{1}+\kappa p_{2}\right) \neq 0$, the divergence cannot occur. Thus, the only bifurcations possible would be dynamic, inducing auto-excited oscillations (Poincaré - Andronov-Hopf bifurcations). On the boundaries of the stability domains, the characteristic exponents are purely imaginary and can be written as $\lambda=2 \pi i s$, with realvalued $s$. One can notice that for integer values of the frequency parameter $s \rho= \pm 1, \pm 2, \pm 3, \ldots$ this equation has no solutions. Then, from Eq. (3.8) we can write the real and imaginary parts on the stability boundaries:

$$
\left\{\begin{array}{l}
\operatorname{Re}[P(i s, \varsigma, \rho, \kappa]=\ldots \\
\ldots=s^{4}-\left(2+\kappa\left(p_{1}+p_{2}\right)+4 \varsigma^{2}\right) s^{2}+\left(1+\kappa\left(p_{1}+p_{2}\right)\right)+\kappa^{2} p_{1} p_{2}(1-\cos (2 \pi \rho s))=0 \\
\operatorname{Im}\left[P(i s, \varsigma, \rho, \kappa]=-4 \varsigma s^{3}+2\left(2+\kappa\left(p_{1}+p_{2}\right)\right) \varsigma s+\kappa^{2} p_{1} p_{2} \sin (2 \pi \rho s)=0 .\right.
\end{array}\right.
$$

Equations (3.9) are determined in parametric form by the boundary of the stability regions for the parameters of relative stiffness constant $\kappa$ and of rotation rate $\rho$.

In the present work Eqs. (3.5) and (3.9) are solved for $\xi_{1}, \xi_{2}, \eta_{1}, \eta_{2}, s, \kappa$ simultaneously, via commonly available routines, such as MATLAB's fsolve. The solution of Eqs. (3.5) and (3.9) leads to the construction of the boundaries of stability regions for the steady continuous cutting regime with respect to parameters $\kappa$ and $\rho$, i.e., respectively, static stiffness cutting and period of rotation. The resulting diagram on the $(\kappa-\rho)$ plane is a stability lobe diagram (SLD). The particularity of the present case is the nonlinear definition of the nominal state, leading to a variation of the effective stiffness coefficients $p_{1,2}$ along the stability boundaries, due to a change in the static deflection magnitude of the cutters $\xi_{1,2}$. Thus, on these boundaries, one can also plot, as a function of dimensionless cutting velocity $1 / \rho$, the respective steady values of axial displacement $\xi_{j 0}$, cutting forces $\Pi_{j 0}$, and cut thickness $\eta_{j 0}$.

\section{Balanced cut thickness distribution case}

An analysis of Eqs. (3.8) shows that at every angular position of cutters $\left(\alpha=\tau_{1} / \tau_{2}\right)$ there exists a value of axial offset $\mathrm{H}_{\alpha}$ leading to equal work of cutters, i.e., $\eta_{10}=\eta_{20}$. In this case of 
balanced cut thickness, we have equal cutting forces $\Pi_{10}=\Pi_{20}$, and therefore, in our system with equal cutter suspension stiffness $k_{1}=k_{2}$, the deflections are also equal, i. e., $\xi_{10}=\xi_{20}$, and finally

$$
\mathrm{H}_{\alpha}=\frac{1-\alpha}{2(1+\alpha)}
$$

It can be observed that this expression, also plotted in Fig. 3, reflects reasonable asymptotic behavior: for symmetric cutters position $(\alpha=1)$ no offset is required $(H=0)$, whereas for very asymmetric cases $(\alpha \rightarrow 0$ or $\alpha \rightarrow \infty)$ half-feed offsets are required $(H \rightarrow \pm 1 / 2)$.

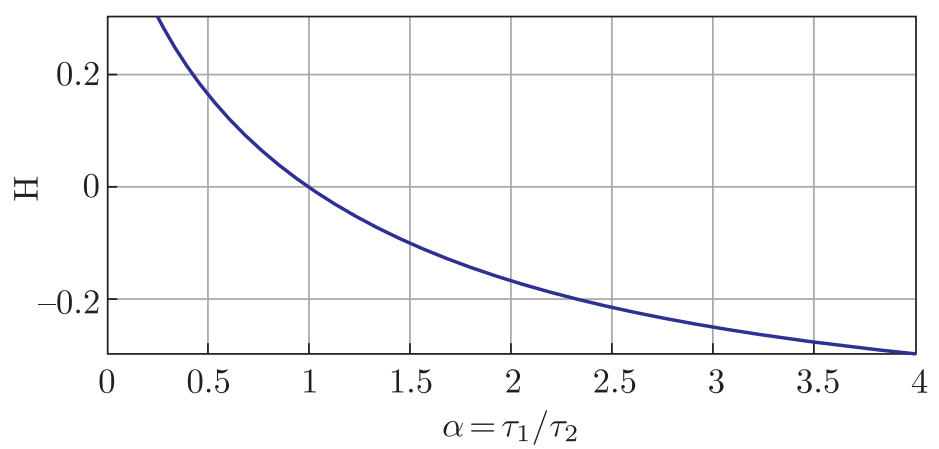

Fig. 3. Angular position vs axial offset for the balanced cut thickness case: $\mathrm{H}_{\alpha}(\alpha)$.

\section{Numerical example}

To illustrate qualitatively the influence of the system configuration on the stability boundaries, we present the four cases summarized in Table 1.

Table 1. Analyzed case

\begin{tabular}{|c|c|c|c|}
\hline Case \# & $\alpha$ & $\mathrm{H}$ & $\kappa$ \\
\hline 1 & 1 & 0 & 0.722 \\
\hline 2 & 1 & 0.1 & 0.361 \\
\hline 3 & 2 & 0 & 0.361 \\
\hline 4 & 1.182 & 0.4 & 0.361 \\
\hline
\end{tabular}

The other parameters defining the systems are identical for all the cases: $\varsigma=0.036, \eta=$ $=0.1, r=0.55$. For each case SLD is computed along with the response magnitude in terms of deflections $\xi$ and cut thicknesses $\eta$ for each cutter.

Figure 4 shows the stability data for cases 1 and 2, featuring the symmetrical angular position of cutters. One can see that in the trivial situation (case 1: no angular or axial offset, Figs. 4a-4c) both cutters work in the same manner. The SLD plots (Figs. 4a, 4d) are composed in a quite classical manner: a set of lobe-shaped stability boundaries with instabilities present above each lobe. Thus, the stable region is situated below all these lines. When comparing 

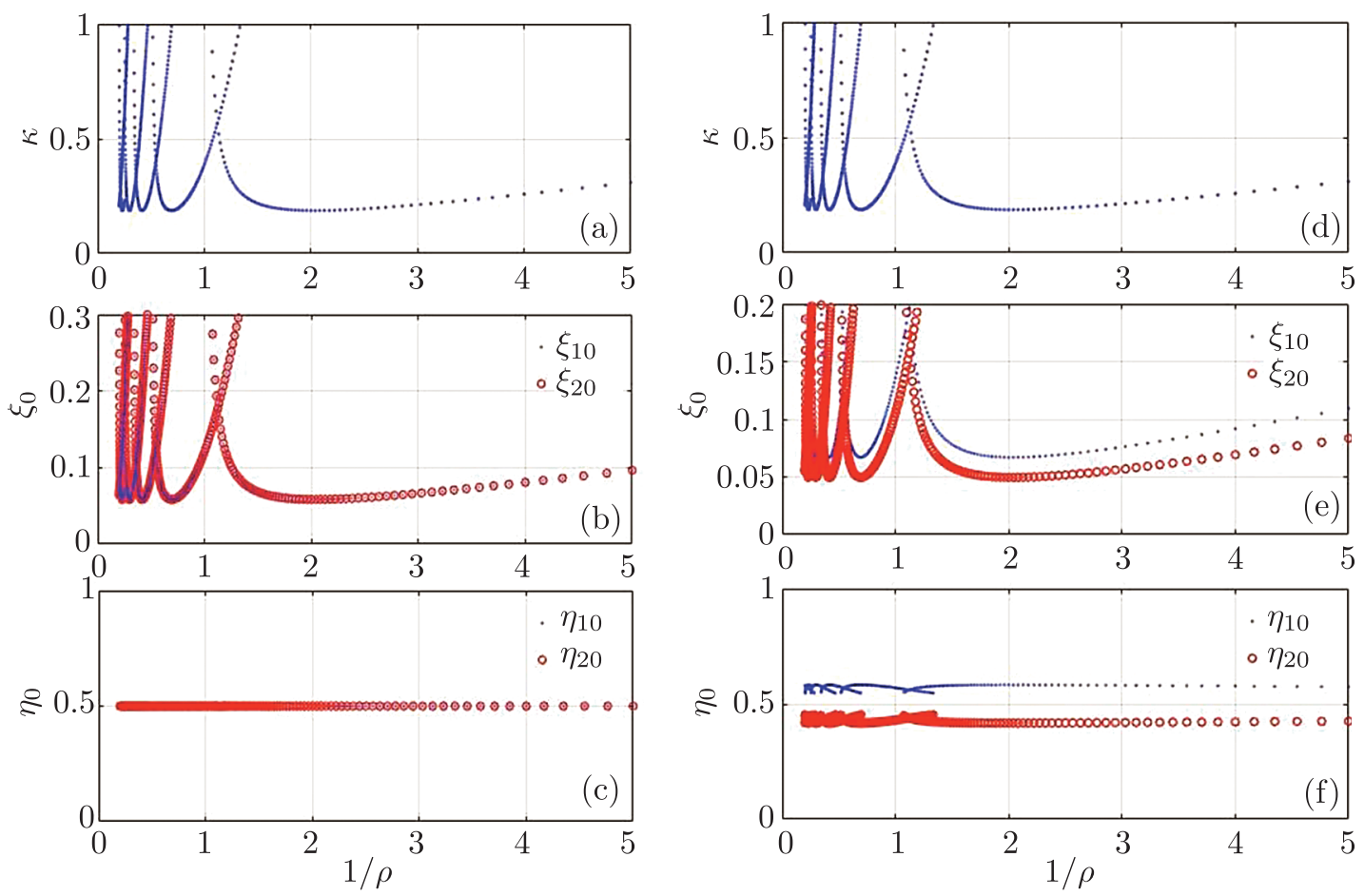

Fig. 4. Stability investigation for symmetrical cutter position $(\alpha=1)$ with zero offset, i.e., $\mathrm{H}=0$ (a)-(c) and nonzero offset, i. e. $\mathrm{H}=0.1(\mathrm{~d})-(\mathrm{f})$ for the case $\eta_{*}=0.1, r=0.55, \varsigma=0.05$ : stability lobe diagram (a), (d) and the steady cutter deflection (b), (e), steady chip thickness (c), (f).

cases 1 and 2, although the SLD are identical, the first cutter is subject to a much stronger deflection $\xi_{1}>\xi_{2}$ (see Fig. 4e) accompanying higher matter removal $\eta_{1}>\eta_{2}$ (see Fig. 4f).

From Fig. 5, comparing two cases of unsymmetrical circumferential arrangement of cutters (cases 3 and 4), one can observe that the SLD features a very weak variation (within $5 \%$ in the rotation rate range considered). On the other hand, response magnitudes are subject to stronger variation between cases 3 and 4 .

\section{Time marching solution involving $\varepsilon$-embedding}

To observe the postcritical behavior of the system in the domain of instability of the steady static cut, we carry out time marching simulation.

Numerical solution of the delayed DDAE system (3.2) is carried out in MATLAB. For this purpose a system of delay differential equations (DDE) was derived from (3.2), via the $\varepsilon$-embedding approach: the algebraic part of the system (3.2) is transformed into differential via an additional perturbation term ( $\varepsilon$-embedding) with a small parameter $\varepsilon$ :

$$
\varepsilon \Lambda_{j}^{\prime}(\tau)=-\Lambda_{j}(\tau)+\Lambda_{j-1}\left(\tau-\tau_{j-1}\right)+\eta_{j}(\tau), \quad 0<\varepsilon \leqslant 1 .
$$

As a result, one obtains a DDE system:

$$
\left\{\begin{array}{l}
\varepsilon \Lambda_{1}^{\prime}(\tau)=-\Lambda_{1}(\tau)+\Lambda_{2}\left(\tau-\tau_{2}\right)+\eta_{1}(\tau), \\
\varepsilon \Lambda_{2}^{\prime}(\tau)=-\Lambda_{2}(\tau)+\Lambda_{1}\left(\tau-\tau_{1}\right)+\eta_{2}(\tau), \\
\xi_{j}^{\prime \prime}=-4 \pi \varsigma \xi_{j}^{\prime}-4 \pi^{2} \xi_{j}+4 \pi^{2} \kappa \Pi_{j} ; \quad j=1,2 .
\end{array}\right.
$$



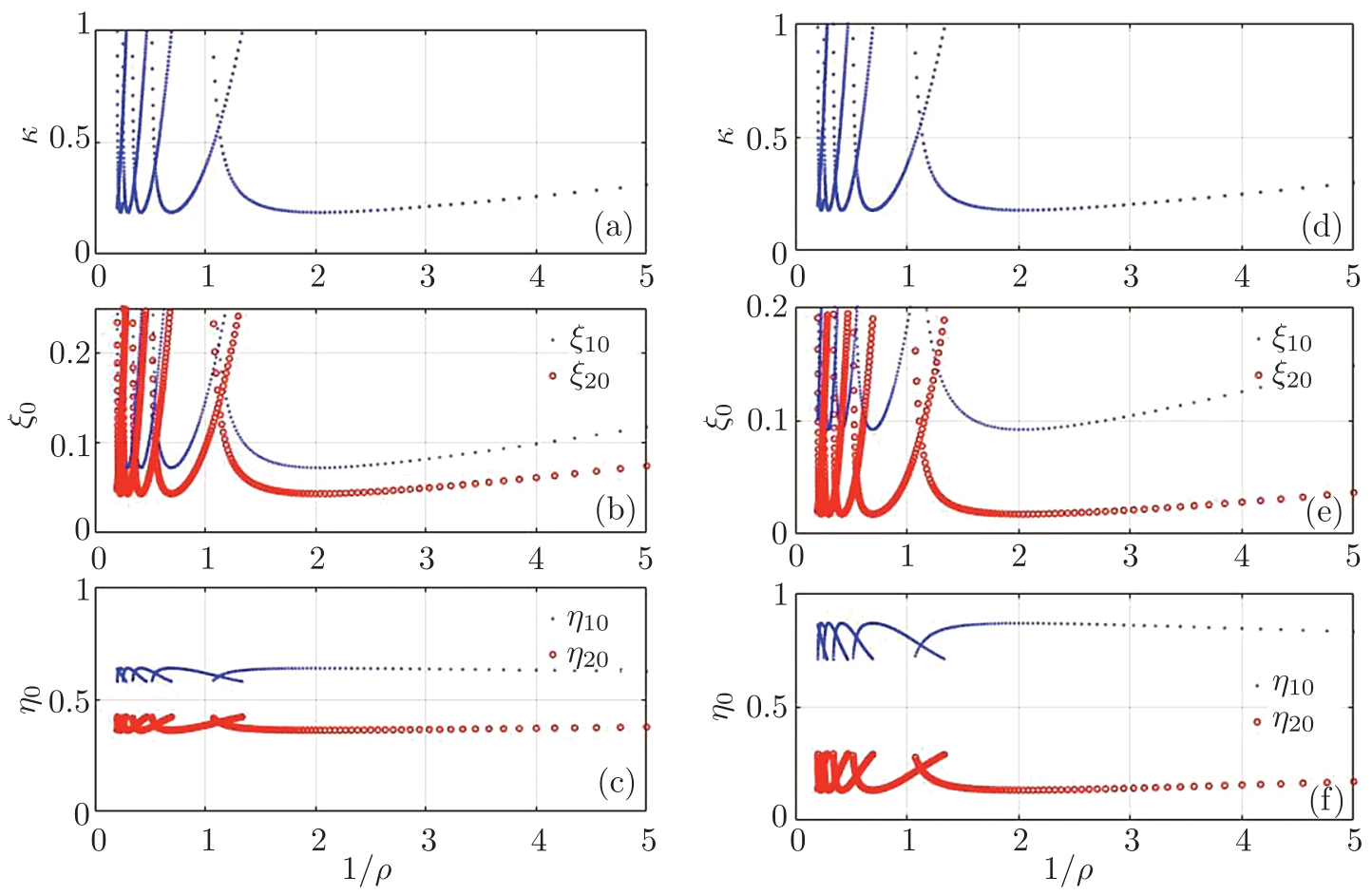

Fig. 5. Stability investigation for nonsymmetrical cutter position and zero axial shift, i.e., $\alpha=2, \mathrm{H}=0$ (a)-(c) and nonzero shift, i. e., $\alpha=1.18, \mathrm{H}=0.4$ (d)-(f) for the case $\eta_{*}=0.1, r=0.55, \varsigma=0.05$ stability lobe diagram (a), (d) and the steady cutter deflection (b), (e), steady chip thickness (c), (f).

with

$$
\left\{\begin{array}{l}
\Delta_{1}(\tau)=\tau / \rho-\xi_{1}(\tau)-\Lambda_{2}\left(\tau-\tau_{2}\right)+A, \\
\Delta_{2}(\tau)=\tau / \rho-\xi_{2}(\tau)-\Lambda_{1}\left(\tau-\tau_{1}\right)+A-\mathrm{H}_{0}, \\
\eta_{j}(\tau)=\max \left[0, \Delta_{j}(\tau)\right] ; \Pi_{j}=\eta_{j} \frac{\eta_{*}+r \eta_{j}}{\eta_{*}+\eta_{j}}, j=1,2 .
\end{array}\right.
$$

The system (3.9) is suitable for processing with MATLAB-based utilities, such as dde23 solver.

Numerical simulations based on the above-mentioned framework have enabled the computation of axial vibrations of the cutters $\xi_{j}$ (Fig. 3) and cut thickness profiles (Fig. 4) as a function of dimensionless time $\tau / \rho$ for cases 1-4 (see Table 1) $\rho=5.94$. All the simulations feature instability of steady cut, with a limit cycle oscillatory response. When an axial offset $\mathrm{H}=0.4$ or an angular asymmetry is present, the vibrations of cutters show slight differences, while important dissimilarity in chip shape and thickness arises (Figs. 6 and 7). On can notice that the cutters engage simultaneously (case 1 - self-synchronization) or alternating (cases 2, 3 and 4). Figures 6 a and 7 a show the case of a symmetrical arrangement of the cutting edges of both cutters $\varphi_{1}=\varphi_{2}=180^{\circ}, \mathrm{H}=0$. In the steady state, both cutters move symmetrically and generate intermittent chips of the same shapes. In the presence of axial shear (Figs. 6c and 7c) the cutters oscillate in antiphase and intermittent chips are generated. Figures $6 \mathrm{c}, 6 \mathrm{~d}$ and $7 \mathrm{c}, 7 \mathrm{~d}$ demonstrate the possibilities of controlling the process of vibrations by choosing different values of axial and angular shift. 


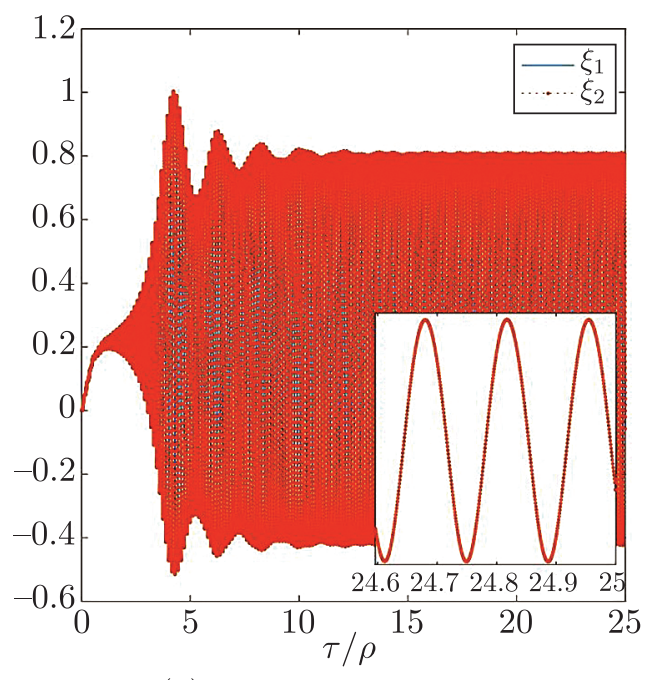

(a) $\varphi_{1}=\varphi_{2}=180^{\circ}, \mathrm{H}=0$

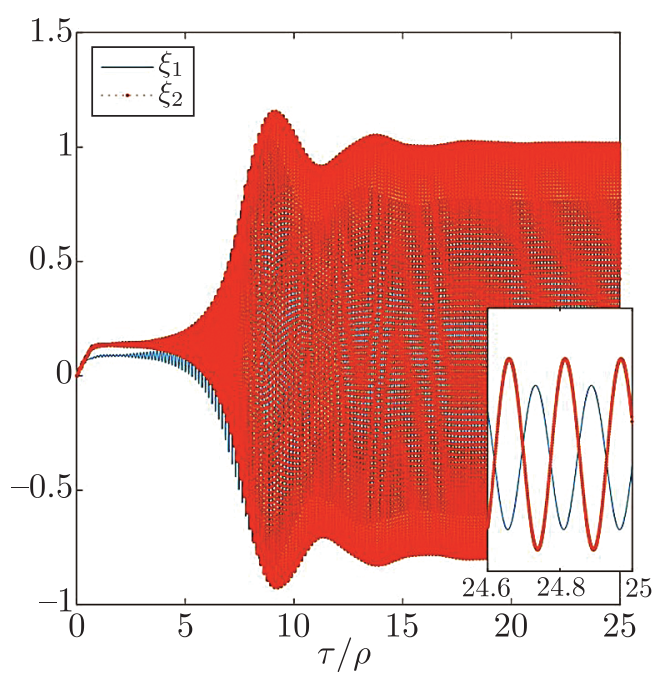

(c) $\varphi_{1}=240^{\circ}, \varphi_{2}=120^{\circ}, \mathrm{H}=0$

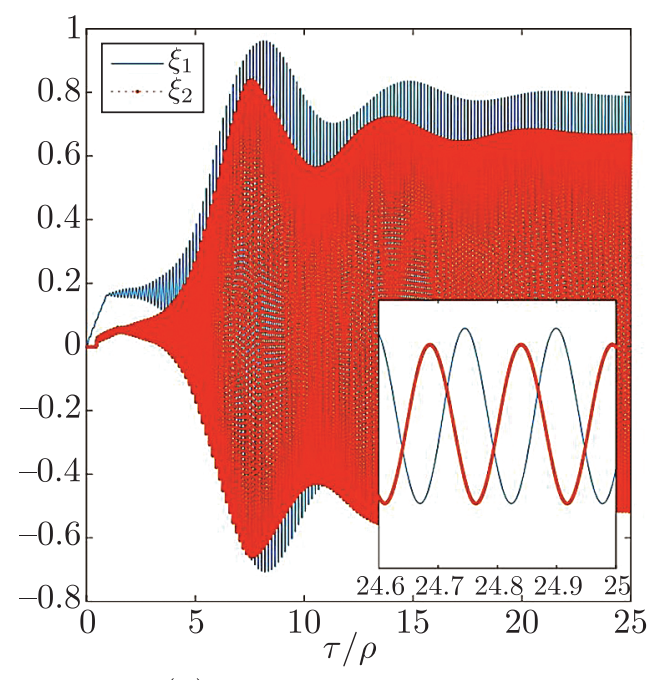

(b) $\varphi_{1}=\varphi_{2}=180^{\circ}, \mathrm{H}=0.4$

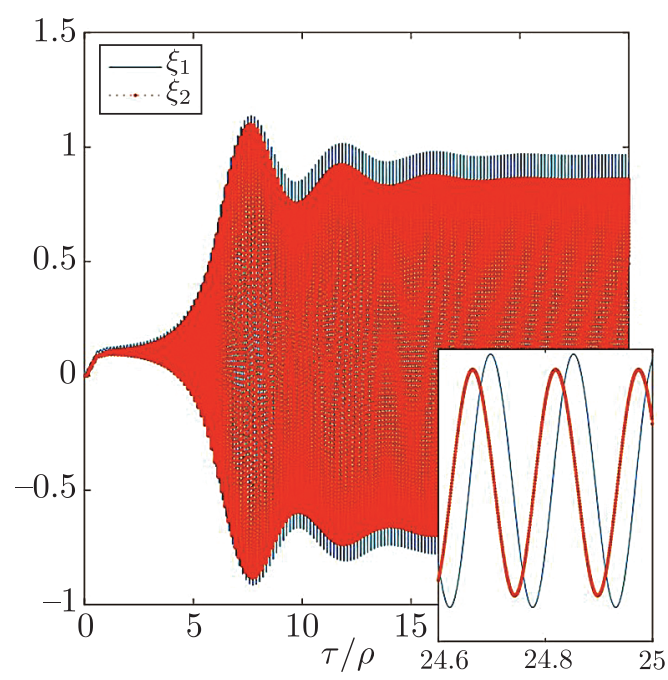

(d) $\varphi_{1}=195^{\circ}, \varphi_{2}=165^{\circ}, \mathrm{H}=0.1$

Fig. 6. Axial oscillations of symmetrical (a), (b) and nonsymmetrical (c), (d) angular position of cutters and for various cases of axial offset $\mathrm{H}$ as a function of nondimensional time $\tau / \rho$.

\section{Conlusion}

A mathematical model of the dynamics of multiple cutter turning has been developed with the regenerative effect taken into account. For numerical realization in MATLAB environment, the delayed DDAE are transformed into DDE by an $\varepsilon$-embedding approach. The analysis of the dynamics of the multicutter turning has revealed the impact of the angular and axial positions of cutting edges on the shape and thickness of the chip removed by each cutter.

It has been shown that the stability boundaries of the steady cutting regime depend on the workpiece revolution period and are independent of the angular positions of the cutters, when the latter are all aligned in the axial direction.

The results obtained make it possible to avoid the occurrence of interrupted cutting when technological machining modes are assigned or, on the contrary, when the use of high-intensity 

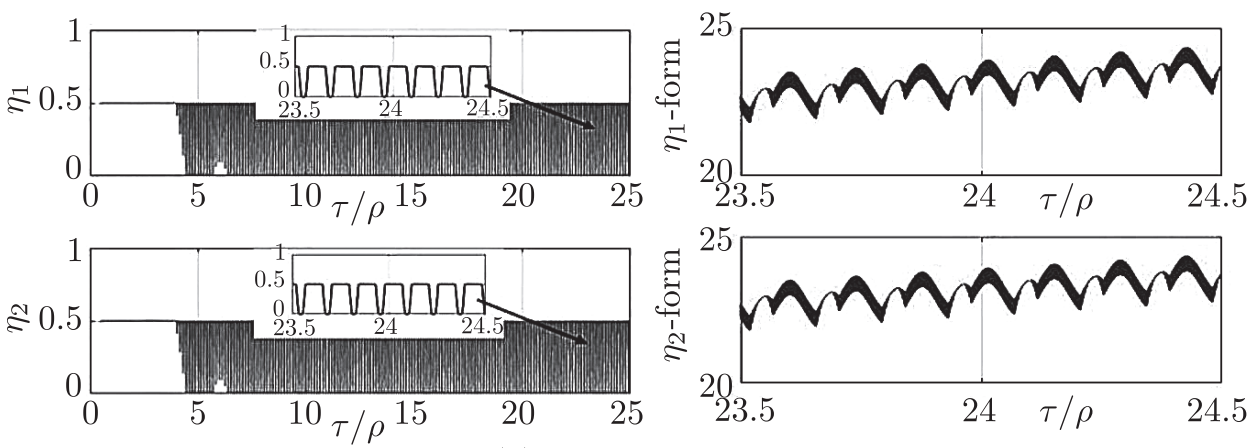

(a) $\varphi_{1}=\varphi_{2}=180^{\circ}, \mathrm{H}=0$
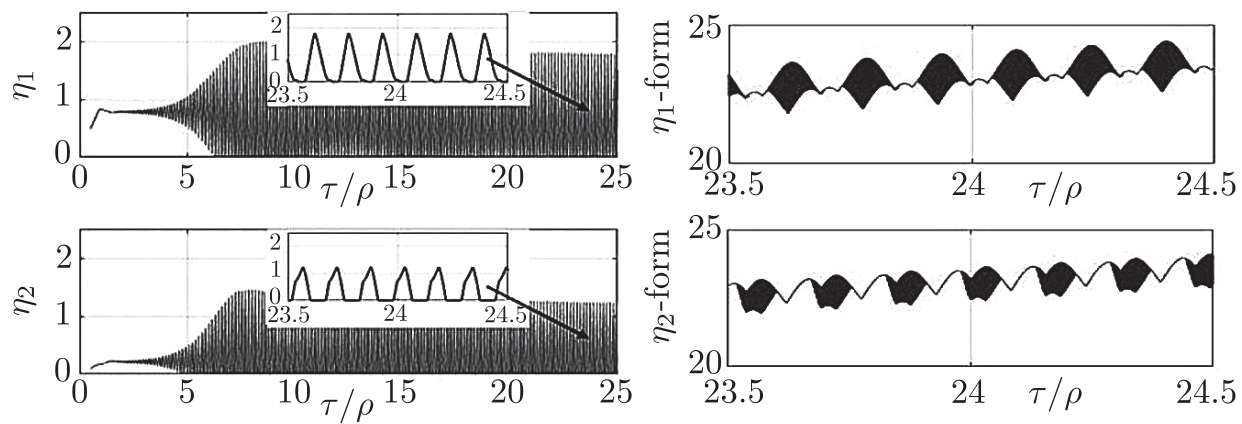

(b) $\varphi_{1}=\varphi_{2}=180^{\circ}, \mathrm{H}=0.4$
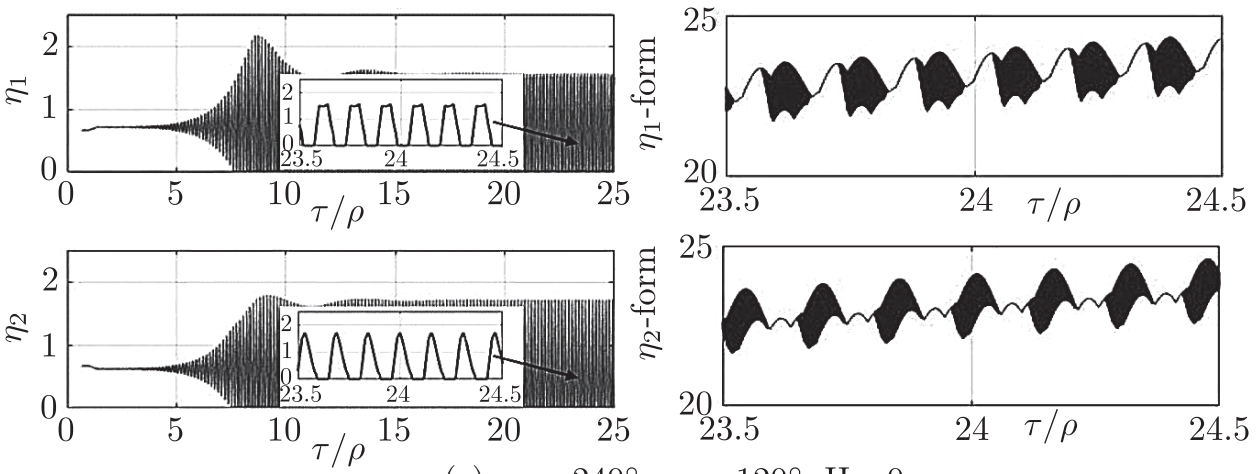

(c) $\varphi_{1}=240^{\circ}, \varphi_{2}=120^{\circ}, \mathrm{H}=0$
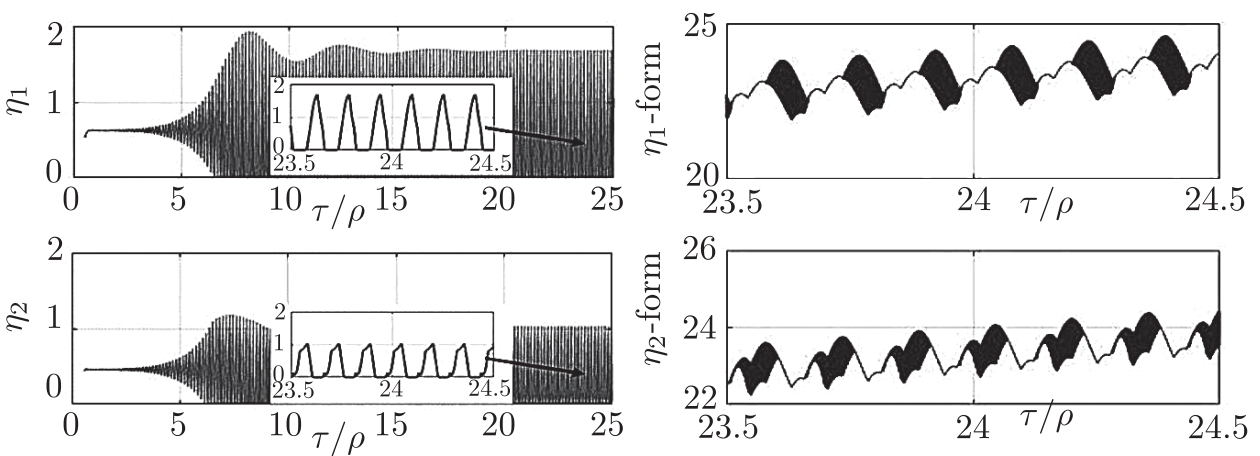

(d) $\varphi_{1}=195^{\circ}, \varphi_{2}=165^{\circ}, \mathrm{H}=0.1$

Fig. 7. Thickness (left) and shape (right) of chips cut by each cutter; (a) symmetric angular position of cutters without axial shift, (b) symmetric angular position of cutters with axial shift, (c) nonsymmetric angular position without axial shift, (d) nonsymmetric angular position with axial shift. 
cutting modes associated with breached chips (for example, during roughing) is necessary, an intermittent cutting mode can be used. This is easily achieved by analyzing the results of nonlinear dynamics and the stability diagrams presented above.

\section{References}

[1] Astashev, V. K. and Korendyasev, G. K., Thermomechanical Model of the Occurrence of Oscillations in Metal Cutting, J. Mach. Manuf. Reliab., 2012, vol.41, no.3, pp.189-193; see also: Problemy Mashinostroeniya i Nadezhnosti Mashin, 2012, no.3, pp. 3-9.

[2] Azvar, M. and Budak, E., Multi-Dimensional Modelling of Chatter Stability in Parallel Turning Operation, in Proc. of the 17th Internat. Conf. on Machine Design and Production (Bursa, Turkey, July 12-15, 2016), $13 \mathrm{pp}$.

[3] Benardos, P. G., Mosialos, S., and Vosniakos, G. C., Prediction of Workpiece Elastic Deflections under Cutting Forces in Turning, Robot. Comput. Integr. Manuf., 2002, vol.22, nos. 5-6, pp. 505514.

[4] Brecher, C., Epple, A., Neus, S., and Fey, M., Optimal Process Parameters for Parallel Turning Operations on Shared Cutting Surfaces, Int. J. Mach. Tool. Manu., 2015, vol. 95, pp. 13-19.

[5] Paris, H., Brissaud, D., Gouskov, A., Guibert, N., and Rech, J., Influence of the Ploughing Effect on the Dynamic Behavior of the Self-Vibratory Drilling Head, CIRP Ann., 2008, vol.57, no.1, pp. 385-388.

[6] Dombovari, Z., Barton, D. A. W., Wilson, R. E., and Stepan, G., On the Global Dynamics of Chatter in the Orthogonal Cutting Model, Int. J. Nonlin. Mech., 2011, vo.l46, no.1, pp. 330-338.

[7] Gerasimenko, A. A., Guskov, M. A., Gouskov, A. M., Lorong, Ph., and Panovko, G. Ya., Analytical Approach of Turning Thin-Walled Tubular Parts. Stability Analysis of Regenerative Chatter, Vibroeng. Proc., 2016, vol. 8, pp. 179-184.

[8] Gouskov, A. M., Voronov, S. A., Paris, H., and Batzer, S. A., Cylindrical Workpiece Turning Using Multiple-Cutting Tool, in Proc. of the Design Technical Conf. and Computers and Information Engineering Conf. (Pittsburgh, Pa., 2001).

[9] Gouskov, A. M., Voronov, S. A., Paris, H., and Batzer, S. A., Nonlinear Dynamics of a Machining System with Two Interdependent Delays, Comm. Nonlinear Sci. Numer. Simulat., 2002, vol. 7, no. 3, pp. 207-221.

[10] Gouskov, A. M., Guskov, M. A., Lorong, Ph., and Panovko, G. Ya., Influence of the Clearance Face on the Condition of Chatter Self-Excitation during Turning, IJMMM, 2017, vol. 19, no. 1, pp. 17-39.

[11] Gouskov, A. M., Guskov, M. A., Ding Dyk Tung, and Panovko, G. Ya., Multi-Cutter Turning Process Stability Analysis, Vibroeng. Proc., 2018, vol.17, pp. 124-128.

[12] Gouskov, A. M., Guskov, M. A., Dinh Duc Tung, and Panovko, G. Ya., Modeling and Investigation of the Stability of a Multicuttet Turning Process by a Trace, J. Mach. Manuf. Reliab., 2018, vol. 47, no. 4, pp.317-323; see also: Problemy Mashinostroeniya i Nadezhnosti Mashin, 2018, no. 4, pp. 1927.

[13] Gouskov, A. M., Dynamics of Two-Cutter Turning: 1, Stanki i Instrument, 2004, no. 11, pp. 3-6 (Russian).

[14] Kalidasan, R., Yatin, M., Sarma, D. K., Senthilvelan, S., and Dixit, U. S., An Experimental Study of Cutting Forces and Temperature in Multi-Tool Turning of Grey Cast Iron, IJMMM, 2016, vol. 18, nos. 5/6, pp. 540-551.

[15] Kondratenko, K., Gouskov, A., Guskov, M., Lorong, Ph., and Panovko, G., Analysis of Indirect Measurement of Cutting Forces Turning Metal Cylindrical Shells, in Vibration Engineering and Technology of Machinery, J. Sinha (Ed.), Mechan. Machine Science, vol. 23, Cham: Springer, 2015, pp. 929937. 
[16] Kozochkin, M.P., Dynamic of Cutting Process: Theory, Experiment, Analysis, Saarbrücken: Lambert, 2013 (Russian).

[17] Kudinov, V.A., Dynamics of Machine Tools, Moscow: Mashinostroenie, 1967 (Russian).

[18] Lamikiz, A., Lopez de Lacalle, L. N., Sanchez, J.A., and Bravo, U., Calculation of the Specific Cutting Coefficients and Geometrical Aspects in Sculptured Surface Machining, Mach. Sci. Technol., 2005, vol. 9, no. 3, pp. 411-436.

[19] Ozturk, E., Comak, A., and Budak, E., Tuning of Tool Dynamics for Increased Stability of Parallel (Simultaneous) Turning Processes, J. Sound Vibration, 2016, vol. 360, pp. 17-30.

[20] Reith, M. J., Bachrathy, D., and Stepan, G., Improving the Stability of Multi-Cutter Turning with Detuned Dynamics, Machining Science and Technology, Mach. Sci. Technol., 2016, vol. 20, no. 3, pp. 440-459.

[21] Wang, X. and Feng, C.X., Development of Empirical Models for Surface Roughness Prediction in Finish Turning, Int. J. Adv. Manuf. Technol., 2002, vol. 20, no. 5, pp. 348-356. 